\title{
Mobile Small RNAs Are Predominately Accumulated via Long-Distance Movement Rather than Local Biogenesis
}

\section{Shuai Li}

Qingdao Agricultural University

\section{Xutong Wang}

Purdue University https://orcid.org/0000-0002-4625-7659

\section{Wenying Xu}

Qingdao Agricultural University

\section{Tong Xu}

Qingdao Agricultural University

\section{Chunmei Cai}

Qingdao Agricultural University

\section{Liyang Chen}

Purdue University https://orcid.org/0000-0002-4102-4354

\section{Chancelor Clark}

Purdue University

Jianxin Ma ( $\square$ maj@purdue.edu )

Purdue University https://orcid.org/0000-0002-1474-812X

\section{Article}

Keywords: microRNAs, mobile RNAs, phasiRNAs, siRNAs, systemic gene regulation

Posted Date: August 5th, 2020

DOI: https://doi.org/10.21203/rs.3.rs-49850/v1

License: (c) (1) This work is licensed under a Creative Commons Attribution 4.0 International License. Read Full License

Version of Record: A version of this preprint was published at Nature Plants on January 15th, 2021. See the published version at https://doi.org/10.1038/s41477-020-00829-2. 
3 Mobile Small RNAs Are Predominately Accumulated via Long-Distance Movement Rather

\section{4 than Local Biogenesis}

6 Shuai Li1,2, $\uparrow$, Xutong Wang $1, \uparrow$, Wenying Xu2, Tong Liu2, Chunmei Cai2, Liyang Chen1, Chancelor

7 B. Clark1, Jianxin Ma1,3*

8

9 1Department of Agronomy, Purdue University, West Lafayette, IN 47907, USA

10 2College of Life Sciences, Qingdao Agricultural University, Qingdao, 266109, China

11 3Center for Plant Biology, Purdue University, West Lafayette, IN 47907, USA

$12 \dagger$ These authors contributed equally to this work

$13 *$ Correspondence Email: maj@purdue.edu

14

15 Key words: microRNAs, mobile RNAs, phasiRNAs, siRNAs, systemic gene regulation 
Long-distance RNA movement is important for plant growth and environmental responses; however, the extent to which RNAs move between distant tissues, their relative magnitude and functional significance remain to be elucidated on a genomic scale. Using a soybean (Glycine max)/common bean (Phaseolus vulgaris) grafting system, we identified 100 shootroot mobile miRNAs and 32 shoot-root mobile phasiRNAs, which were predominantly produced in shoots but transported to roots, some of which enabled cleavage of their mRNA targets or their precursors. In contrast, most of the mobile mRNAs were transcribed in both shoots and roots and were truncated fragments, with the transported copies accounting for only a tiny portion of all copies accumulated in the recipient (shoots or roots) tissues. These findings suggest that the regulatory mechanisms for sRNAs movement are different from those for mRNA movement, and that the earlier is more strictly regulated, and likely, more functionally significant than the latter. Introduction Higher plants possess two specialized vascular tissues - the xylem and phloem, which conduct water and nutrients from the roots to the shoots and organic components produced by 

xylem, which is composed of primarily dead cells, the phloem consists of columns of living cells, including sieve elements and companion cells connected by plasmodesmata, allowing molecules

37 such as carbohydrates, proteins/amino acids, and hormones to travel locally or over long

38 distances $1,2,3,4,5$. The phloem also harbors a variety of RNAs such as messenger RNAs (mRNAs), miRNAs, and small interfering RNAs (siRNAs) 1,2,3,6,7. Early studies demonstrated that several mRNAs produced in leaves were transported to distal tissues to exert physiological functions underlying specific traits $8,9,10,11,12$. Recent genomic analyses of transcripts in heterografted plants

42 identified hundreds to thousands of mRNAs involved in shoot-to-root or vice versa 43 trafficking $1,2,3,13$. In the past decade, several miRNAs have been identified as systemic signals

44 mediating long-distance communications 14,15,16,17. For example, miR399 in Arabidopsis thaliana and miR395 in Brassica rapa move from shoots to roots in response to nutrient deficiencies, while

$46 \mathrm{miR} 2111$ in Lotus japonicus travels from shoots to roots to regulate the susceptibility of uninfected

47 roots to the soil bacteria rhizobia as a mechanism to balance rhizobial infection and nodulation events 18,19,20,21. More recently, a genomic analysis of grafted tissues identified numerous transposon-derived or heterochromatic siRNAs (hcsiRNAs) that are capable of trafficking from

50 shoots to roots to modulate genome-wide DNA methylation in the recipient root cells22,23.

51 Despite such a progress, surprisingly, genome-scale identification and characterization of 52 mobile miRNAs - important regulators of gene expression - has not been conducted in any 

organisms. Phased secondary siRNAs (phasiRNAs), a subclass of siRNAs that require a trigger miRNA for their biogenesis, were known to be capabale of cell-to-cell trafficking24,25,26, but

55 whether they move over long distance is yet to be determined. Shoots-to-roots mobile hcsiRNAs

56 have been identified at the whole genome level, but whether hcsiRNAs also move from roots to

57 shoots to regulate DNA methylation has not been investigated. Long-distance mobile mRNAs have

58 been identified on a genome scale in several plants, but the integrity and abundance of mobile

59 mRNAs in recipient tissues relative to those produced locally are unclear. Here, we integrate

60 sRNA-seq, Illumina short-read RNA-seq, Nanopore long-read RNA-seq, and degradome-seq data

61 from heterografted and autografted soybean (Glycine max) and common bean (Phaseolus vulgaris)

62 - two economically important leguminous crops diverged from a common ancestor $\sim 17$ million

63 years ago - to address these outstanding questions.

64

65 Results

66

Identification of mobile sRNAs using heterografted and autografted plants. To identify mobile

67 RNAs and reveal the extent to which they move over long distance, we conducted grafting

68 experiments that involved four distinct grafted scion/rootstock combinations - soybean/common

69 bean and common bean/soybean heterografted plants, and soybean/soybean and common 70 bean/common bean homografted plants. Grafting was conducted at the V1 developmental stage of 
71

72

73

74

75

76

77

78

79

80

81

82

83

84

85

86

87

88

soybean plants when their first set of unfolded trifoliate leaves emerged, then four shoot samples and four root samples were collected 10 days after grafting for RNA isolation and sequencing (Fig.

1a; see details in Methods). A total of 75,512 unique sRNAs ranging from 16 to 26 -nt in size were obtained from the eight samples. Of these, 21,141 (28.0\%) were from soybean (Gm-sRNAs), whereas 19,885 (26.3\%) were from common bean (Pv-sRNAs). The origins of the remaining sRNAs could not be determined as they were perfectly mapped to both the soybean and common bean reference genomes27,28 (Fig. 1b, Supplementary Dataset1). Comparison of the shoot and root samples from the heterografted plants (see details in Methods) revealed 4,223 Gm-sRNAs and 7,063 Pv-sRNAs moving from shoots to roots, four Pv-sRNAs moving from roots to shoots, and one Gm-sRNA moving bidirectionally (Fig. 1c, Supplementary Table 1). These mobile sRNAs together, account for $27.5 \%$ of all the sRNAs distinguishable between the two crops.

Long-distance movement and relative abundance of hcsiRNAs. In either soybean or common bean, the 24-nt sRNAs are most abundant and match primarily to transposable elements27,28, and were considered as hcsiRNAs (Fig. 1d). Of the 4,224 mobile Gm-sRNAs, 1,214 (28.7\%) are GmhcsiRNAs, which showed mobility from shoots to roots. Of the 7,067 mobile Pv-sRNAs, 4,018 (56.9\%) are Pv-hcsiRNAs, which were capable of moving from shoots to roots. None of the GmhcsiRNAs or Pv-hcsiRNAs was detected to have moved from roots to shoots (Supplementary 
Table 1). Of the 1,214 shoot-to-root mobile Gm-hcsiRNAs and 4,018 shoot-to-root mobile PvhcsiRNAs, 1,146 (94.4\%) and 3,883 (96.6\%) were not detected in the heterografted soybean roots

91 and heterografted common bean roots, respectively (Fig. 1e,f and Supplementary Table 2). Even

92 though the remaining $68 \mathrm{Gm}$-hcsiRNAs and $135 \mathrm{Pv}$-hcsiRNAs were detected in the heterografted soybean roots and heterografted common bean roots, their relative abundances were substantially

94 lower than detected in the homografted soybean roots and homografted common bean roots, respectively, indicating that mobile hcsiRNAs are predominantly produced in shoots instead of

96 roots (Fig. 1e,f and Supplementary Table 2). Overall, the relative abundance of the mobile Gm-

97 hcsiRNAs in heterografted common bean roots was similar to that observed in homografted soybean roots and vice versa (Fig. 1g,h), suggesting that the biogenesis and mobility of the hcsiRNAs were likely not affected by the recipient tissues.

100

Long-distance movement, relative abundance, and miRNA-mediated systemic regulation. A total of 622 miRNAs were identified in the eight samples, including $161 \mathrm{Gm}$-miRNAs, 72 PvmiRNAs, and 389 miRNAs sharing identical sequences between soybean and common bean. Of the $161 \mathrm{Gm}$-miRNAs, 67 were detected to be mobile from shoots to roots, one was detected to be mobile bidirectionally, and the remaining 93 were immobile between shoots and roots. Of the 72 
107 shoots and roots (Supplementary Table 1). Of the 67 shoot-to-root mobile Gm-miRNAs and 33 108 shoot-to-root mobile Pv-miRNAs, $63(94.0 \%)$ and $25(75.8 \%)$ were not detected in the 109 heterografted soybean roots and heterografted common bean roots, respectively (Fig. 2a,b and 110 Supplementary Table 3). Of these mobile miRNAs, six (miR166i, miR1509a, miR1510a, $111 \mathrm{miR} 1510 \mathrm{~b}, \mathrm{miR} 5770 \mathrm{a}$, and miR5770b) were detected to have moved from shoots to roots in both 112 soybean and common bean (Supplementary Table 4). Overall, the relative abundance of the mobile

113 Gm-miRNAs in heterografted common bean roots was similar to that observed in homograft

114 soybean roots and vice versa, but exceptions were also observed (Fig. 2c,d and Supplementary

115 Table 3). The mobility of several miRNAs was validated by stem-loop PCR (Supplementary Fig.

116 1). In contrast to the mobile miRNAs, their precursors were expressed at similar levels between

117 the homografted roots and heterografted roots of a same species (Fig. 2e,f). These observations

118 indicate that the mobile Gm-miRNAs were predominantly produced in shoots, and accumulated

119 in heterografted roots to a level that is similar to what was detected in the homografted roots of the

120 same species, although there are exceptions, particularly, for some of the Pv-miRNAs (Fig. 2g).

121 Of the 67 mobile Gm-miRNAs and 33 mobile Pv-miRNAs, 32 and 10 were detected to enable

122 the cleavage of their putative mRNAs in either soybean, or common bean, or both by degradome-

123 seq (Supplementary Table 5). When RNA-seq data from the eight tissues were integrated, five

124 soybean and four common bean genes, whose mRNAs were cleaved by corresponding common 
125 bean and soybean mobile miRNAs, showed a reduced level of expression in heterografted soybean

126 and common bean roots, respectively. By contrast, such cleavages and reduction in mRNA

127 abundance were not detected in homografted soybean and common bean roots. In addition, 12

128 soybean genes were detected to be targeted by soybean miRNAs and meanwhile exhibit reduced

129 expression in homografted soybean roots compared with heterografted soybean roots, in which no

130 cleavages of those mRNAs were detected (Fig. 2h,i,j). One of the mobile miRNAs, gma-

$131 \mathrm{miR} 4415 \mathrm{a} / \mathrm{b}-3 \mathrm{p}$, which was produced in soybean shoots and moved to the homografted soybean

132 roots and heterografted common bean roots was able to target soybean gene Glyma.20G051900

133 and common bean gene Phvul.006g011700, respectively, resulting in reduced expression of

134 respective targets in the two roots.

Long-distance movement, relative abundance, and phasiRNA-mediated systemic regulation.

137 Based on the degradome-seq and sRNA-seq data, a total of 29 soybean $P H A S$ loci and 13 common

140 between soybean and common bean. Of the $61 \mathrm{Gm}$-phasiRNAs, 23 were detected to move from

141 shoots to roots, and 38 were immobile. Of the 18 Pv-phasiRNAs, 14 were detected to move from

142 shoots to roots and 4 were immobile (Fig. 3a,b and Supplementary Table 6). The relative 

abundance of the mobile Gm-phasiRNAs in heterografted common bean roots was similar to that

144 observed in homografted soybean roots and vice versa (Fig. 3c,d and Supplementary Table 6). Few

145 of these mobile Gm-phasiRNAs and Pv-phasiRNAs were detected in heterografted soybean and

146 common bean roots, although their phasiRNA precursor transcripts showed similar levels of

147 abundance in heterograft and homograft roots of either soybean or common bean (Fig. 3a,b,e,f and

148 Supplementary Table 6). These observations suggest that the phasiRNAs were nearly exclusively

149 produced in the shoots instead of in the roots (Fig. 3h). Biogenesis of phasiRNAs from their

150 precursor transcripts (“PHAS" loci) requires trigger miRNAs. As exemplified in Figure 3g, gma-

151 miR1510b-3p was detected to have triggered the production of phasiRNAs from the transcripts of

152 Glyms.04G219600 and Glyma.15G232600 in soybean shoots but no such phasiRNAs were

153 detected in heterografted soybean roots, in which both gma-miR1510b-3p and the transcripts of

154 two soybean genes were present. Degradome-seq revealed that three of the mobile phasiRNAs

155 produced from their precursor - the transcripts of Glyms.04G219600, enabled cis-directed

156 cleavage of the precursor in the homografted soybean roots, and one of the three sites were also

157 further confirmed by 5' Rapid Amplification of cDNA Ends (RACE)-PCR. By contrast, such

158 cleavage sites were not detected in the heterografted soybean roots (Fig. 3g,h), exemplifying

159 phasiRNA-mediated systemic regulation of their own precursors.

160 According to the degradome-seq data, mRNAs from 19 genes were detected to be the targets 
161 of 17 phasiRNAs including four mobile Gm-phasiRNAs, one mobile Pv-phasiRNA, seven

162 immobile Gm-phasiRNAs, and five phasiRNAs that were not distinguishable between the two

163 plants (Supplementary Table 7). Of the four mobile Gm-phasiRNAs, one was able to target two

164 soybean genes (Glyma.16G050500, Glyma.19G100200) in homografted roots and a common bean

165 gene (Phvul.001G087000) in heterografted roots, and three were detected to target four soybean

166 genes in homografted roots. The mobile Pv-phasi-RNA was detected to target a common bean

167 gene (Phvul.001G087000) in homografted roots and a soybean gene (Glyma.19G100200) in

168 heterografted roots. As exemplified in Figure 3h, some phasiRNAs were not only able to target

169 their precursor PHAS loci, but also non-phasiRNA-producing genes.

171 Long-distance movement, relative abundance, and integrity of mobile mRNAs. Thousands of

172 plant mRNAs capable of moving between shoots and roots have been previously identified through

173 RNA-sequencing of grafted tissues in multiple heterograft systems; however, large proportions of

174 short RNA-seq reads from heterografted species were unassignable to either species, thus, the

175 integrity of the mobile mRNAs remains largely unknown. In an attempt to assess the relative

176 completeness of mobile mRNAs, we decoded the transcriptomes of the eight grafted samples by

177 Illumina short-read RNA-seq and Oxford Nanopore long-read RNA-seq, which together detected

178 expression of 38,241 soybean genes and 24,062 common bean genes. The short-read RNA-seq 
179 data revealed the mobility of 1,322 mobile soybean mRNAs and 874 common bean mRNAs. Of 180 the 1,322 soybean mRNAs, 1,167 moved from shoots to roots, 130 moved from roots to shoots, 181 and 25 moved bidirectionally. Of the 874 common bean mRNAs, 684 moved from shoots to roots,

182153 moved from roots to shoots, and 37 moved bidirectionally (Fig. 4a and Supplementary Table

1831 and 8). The long-read RNA-seq data revealed the mobility of 163 soybean mRNAs and 129

184 common bean mRNAs, of which, $\sim 56 \%$ were detected to cover the "full-length" of respective 185 coding sequences) (Supplementary Table 8). Of the 163 soybean mRNAs, 117 moved from shoots 186 to roots, 35 moved from roots to shoots, and 11 moved bidirectionally. Of the 129 common bean 187 mRNAs, 90 moved from shoots to roots, 29 moved from roots to shoots, and 10 moved 188 bidirectionally. Totaling five pairs of orthologous genes in soybean and common bean were 189 detected to have produced mobile mRNAs in both species. The trafficking of mRNAs from a 190 number of genes including Glyma.11G114000, Glyma.11G228000, Phvul.001G259000, 191 Phvul.001G113800, Phvul.001G229500, Phvul.010G023000 and Phvul.008G285100, which are 192 predicted to be involved in various signaling pathways underlying plant growth, photosynthesis, 193 and/or vesicle-mediated transport, was further confirmed by qRT-PCR (Supplementary Fig. 2).

194 Remarkably, only eight mobile soybean mRNAs and 14 mobile common bean mRNAs were 195 detected by both the short-read RNA-seq and long-read RNA-seq. 
197 ago, leaving $~ 16,500$ duplicated gene pairs retained in the current genome. We found that, of the 198 1,477 mobile mRNAs in soybean, 300 were from singletons, 1,075 from one copy of duplicated 199 gene pairs, and 51 from both copies of duplicated gene pairs (Supplementary Table 9). Thus, no 200 apparent biases of the mobility against either duplicates or singletons were observed $(3.6 \%$ in 201 duplication vs $2.0 \%$ in singleton). Among all mobile mRNAs detected in soybean and common 202 bean, only 72 were from orthologous genes in the two species, although enriched biological 203 pathways of the mobile mRNAs in the two species are similar (Supplementary Fig. 3 and 204 Supplementary Table 10). In contrast to the predominant accumulation of sRNAs in the recipient tissues (mainly roots), 206 the relative abundance of mobile mRNAs in the recipient tissues (both shoots and roots), as 207 detected by comparison between the heterografted and homografted tissues, was extremely low 208 (Fig. 4b and 4c). For example, most of the soybean mRNAs detected in heterografted common 209 bean roots showed high levels of abundance in both homografted soybean roots and heterografted 210 soybean roots (Supplementary Table 8), whereas most of the soybean sRNAs detected in 211 heterografted common bean roots were not detected in the heterografted soybean roots 212 (Supplementary Table 3). These observations indicate that most mobile mRNAs detected in the 213 recipient tissues (either shoots or roots) were actually produced locally, but most mobile sRNAs 
214 accumulated in the roots were transported from shoots.

\section{Discussion}

217 Accumulating evidence has demonstrated the importance of plant mobile RNAs as signal

218 molecules in shoot-root communications, but no previous studies have investigated the mobility

219 of multiple types of RNAs simultaneously at the whole genome level, perhaps, partly due to the

220 limitations of the grafting systems used in those studies. For example, grafting of the wild-type

221 Arabidopsis shoots with the roots of Arabidopsis mutants lacking the functional RNA polymerase

222 IV required for 24-nt sRNA biogenesis identified mobile 24-nt hcsiRNAs29,30, but it would be

223 unable to detect other types of mobile RNAs. Similarly, grafting of different Arabidopsis ecotypes

224 was able to identify a large number of mobile mRNAs7, but it would be ineffective in detecting

225 mobile sRNAs. Although a number of heterografting systems with highly diverged plant species

226 were used to identify genome-wide mobile mRNAs, the effectiveness of those systems in

227 identifying mobile sRNAs was not explored. In our study, the high degree of sequence divergence

228 between the two leguminous crops enabled identification of 5,232 mobile hcsiRNAs, 100 mobile

229 miRNAs, and 41 mobile phasiRNAs, as well as 2,466 mobile mRNAs throughout the two genomes.

230 Given that less than a dozen mobile miRNAs and phasiRNAs have been previously reported3, the

231 effectiveness of our experiments in identifying mobile sRNAs, particularly miRNAs and 
232 phasiRNAs, is laudable. We would like to point out that our approach also has its own limitations.

233 For example, it was only able to determine the mobility of 233 miRNAs that are unambitiously

234 assigned to the soybean or common bean genomes, which account for $\sim 37 \%$ of all the miRNAs

235 detected in the investigated shoot and root tissues. Whether this subset of miRNAs is representative

236 of all the miRNAs expressed in the two crops remains unclear. However, several individual

237 miRNAs showing sequence polymorphisms between soybean and common bean and shoot-to-root

238 trafficking are also shared by other legumes, in which they have been demonstrated to play

239 important roles in root and nodule development. For example, in Medicago truncatula, miR166

240 and miR1509 were found to regulate the root and nodule development by targeting mRNAs

241 encoding HD-ZIP transcription factors and by triggering the production of phasiRNAs from

242 transcripts of an APETALA2 homolog, respectively,31,32. In addition, miR1510 was found to be a

243 young miRNA specific to the Phaseoleae tribe of legumes and a predominant trigger for the

244 production of phasiRNA from transcripts of $N B-L R R$ genes that may underlie plant's responses to

245 biotic stresses31,33,34. Thus, although these miRNA sequences are diverged among species, they

246 appear to be functionally conserved among these legumes and execute their functional roles

247 systemically through long-distance trafficking.

248 It is noteworthy that our study revealed several contrasting features between mobile sRNAs

249 and mobile mRNAs. One of such features is the directionality of RNA movement. The mobile 
250 sRNAs, including hcsiRNAs; miRNAs; and phasiRNAs, were detected to move from shoots to

251 roots only, with few exceptions, whereas $\sim 13 \%$ of the mobile mRNAs were detected to move from

252 roots to shoots. Movement of mRNAs from shoots and roots and vice versa was commonly

253 observed in other grafting experiments, but the numbers of mobile mRNAs as well as the

254 proportions of mobile mRNAs trafficking in the two directions vary greatly among different

255 experiments 35. Such variation may be associated with growth conditions and developmental stages

256 of the grafted plants, the time points after grafting for tissue collection, and sequencing coverages 3.

257 Another feature is the relative abundance of transported RNA copies versus all copies accumulated

258 in the recipient tissues. In general, the mobile sRNAs transported to the recipient tissues (i.e., roots)

259 were highly abundant, and not produced locally, whereas the mRNAs transported to the recipient

260 tissues (either shoots or roots) were detected to be minimal and account for small proportions of

261 all the mRNAs from the same set of genes detected in the same tissues. This may explain why only

262 a small portion of mobile mRNAs were detected by both the short-read sequencing and long-read

263 sequencing of mRNAs. Lastly, a subset of the mobile sRNAs such as miRNAs and phasiRNAs

264 have been validated to play regulatory roles in the recipient tissues, whereas most of the mobile

265 mRNAs were truncated fragments, which apparently cannot be translated into functional proteins.

266 Together, these features suggest that the long-distance trafficking of sRNAs is more strictly

267 regulated than that of mRNAs. Given such a high level of species-specificity and low level of 
268 abundance, most of the mobile mRNAs detected in this and other studies3,35 are likely non-

269 functional in their recipient tissues.

271 Methods

272 Plant materials and growth conditions. Seeds of soybean variety Williams 82 and common bean

273 variety Jiulibai were soaked in water for one day and then sowed in the soil to grow in the growth

274 chamber under the cycles of $12 \mathrm{~h}$ light at $28^{\circ} \mathrm{C} / 12 \mathrm{~h}$ darkness at $24{ }^{\circ} \mathrm{C}$, with humidity at $30 \%$.

275 The stems of the seedlings were cut seven days (V0 stage) after sowing for the grafting experiments

276 following a protocol described previously36. The grafting experiments include four distinct

277 scion/rootstock combinations - soybean/common bean and common bean/soybean heterografted

278 plants, and soybean/soybean and common bean/common bean homografted plants, from which

279 eight tissues, homografted G. max (soybean) shoots (hoGmSt), homografted G. max roots

280 (hoGmRt), heterografted G. max shoots (heGmSt), heterografted G. max roots (heGmRt),

281 homografted $P$. vulgaris (common bean) shoots (hoPvSt), homografted $P$. vulgaris roots (hoPvRt),

282 heterografted $P$. vulgaris shoots (hePvSt), and heterografted $P$. vulgaris roots (hePvRt) were

283 sampled for sequencing and PCR analyses. 

isolated from grafted shoots and roots samples using TRIzol Reagent (Invitrogen/Life

287 Technologies, CA). The sRNA-seq libraries were constructed using the NEBNext ${ }^{\circ}$ Multiplex

288 Small RNA Library Prep Set for Illumina ${ }^{\circledR}$ (NEB, USA.) following the manufacturer's manual, 289 and then sequenced using the Illumina Hiseq 2500 platform to generate the 50-bp single-end reads.

290 The raw reads were processed with the fastx-toolkit (version 0.0.14, 291 http://hannonlab.cshl.edu/fastx_toolkit) for removal of low-quality reads and adaptor sequences, 292 and the processed reads were mapped to the soybean and common bean reference genomes 293 (version 12.1, phytozome) using the Bowtie program37 (version 1.1.1) with 0 mismatches (-v 0). 294 The abundance matrix of sRNAs was normalized to CPM (counts per million reads) based on the 295 mapping results using in-house Perl scripts. Total RNAs were processed using the Epicentre Ribo-zero ${ }^{\text {тм }}$ rRNA Removal Kit (Epicentre, 297 USA) to deplete ribosomal RNAs, and the processed RNA samples were used to construct RNA298 seq libraries using the NEBNext ${ }_{\circledast}$ Ultra ${ }^{\text {тм }}$ Directional RNA Library Prep Kit for Illumina ${ }^{(N E B,}$ 299 USA). Then the RNA libraries were sequenced using the Illumina Hiseq 4000 platform to generate $300150 \mathrm{bp}$ paired-end reads. The raw reads were processed also with the fastx-toolkit program for 301 removal of low quality reads and adaptor sequences, and the processed reads from each libraries 302 were subsequently mapped to the soybean or common bean reference genomes27, 28 using 
STAR38(version 2.5.4b) with the default parameters. The reads uniquely mapped to individual

304 genes were extracted using Samtools39 (version 1.8), and then the abundance of mRNAs from each

gene was counted and normalized to CPM using the Bedtools program 40 (version 2.29.0) and the

306 "EdgeR" packages in R41 (version 3.28), based on the recommendation by its User's Guide for

307 experiments without biological replicates.

Barcoding Kit (SQK-PBK004), following the manufacturer's manuals. The base-called reads

311 were collected and converted to FASTA format using the MinKNOW software

312 (https://nanoporetech.com). The Nanopore reads were mapped to the soybean and common bean

313 genomes using the minimap2 program42 (version 2.11) with the parameters (-ax splice -uf -k14 --

314 secondary $=$ no -- splice-flank=no). The gene expression level was quantified using Stringtie and

315 Cuffmerge (https://www.biostars.org).

317 5'RACE with high-throughput short-read sequencing method for target mRNA confirmation and

318 cleavage site detection, and was conducted following the protocol described previously43 with

319 modifications made by LC-BIO (Hangzhou, China). The 47-bp single-end PARE sequences were

320 sequenced using the Illumina Hiseq 2500 platform. A combination of the degradome-seq data, with 
321 the transcriptome data and the sRNA reads from soybean and common bean were used to detect

322 cleavage sites within mRNAs using the Paresnip2 program44 (version 4.5).

324 Sequence analyses for detection of mobile RNAs. sRNAs with sizes ranging from $16 \mathrm{nt}$ to $26 \mathrm{nt}$

325 and copy numbers $\geq 1 \mathrm{CPM}$ in at least one of the eight samples were kept for detection of mobile

326 sRNAs. The 24-nt sRNAs were defined as hcsiRNAs, the miRNAs were identified by searching

327 against miRbase - the microRNA database 45 , with a focus on previously identified soybean and

328 common bean miRNAs33. The phasiRNAs precursor genes and phasiRNAs were identified using

329 the PhaseTank program46 (version 1.0) with two reference genomes (soybean and common bean)

330 and other default parameters. Putative miRNA triggers for production of phasiRNAs were

331 predicted using degradome data and psRNAtarget - a plant small RNA target analysis server47.

332 mRNAs were identified by comparison with annotated genes in the soybean and common bean

333 genomes27,28. The variety of mobile RNAs showing distinguishable sequences between the two

334 crops were identified following the criteria described below: i) Soybean RNAs (i.e., Gm-RNAs

335 including Gm-hcsiRNAs, Gm-miRNAs, Gm-phasiRNAs, and Gm-mRNAs) were defined to be

336 mobile from shoots to roots when they were detected in hoGmRt, hoGmSt, heGmSt and hePvRt,

337 but not detected in hoPvSt and hoPvRt; ii) Gm-RNAs were defined to be mobile from roots to

338 shoots when they were detected in hoGmRt, hoGmSt, heGmRt and hePvSt, but absent in hoPvSt 

soybean/common bean tissues. PCR, stem-loop qRT-PCR, and 5'RACE-PCR were performed as previously described48,49. In

354 PCR, in which the soybean gene GmCons4 (GenBank ID BU578186)50 and common bean gene

355 PvActin11 (Phvul.008G011000)51 was used as an respective internal reference to quantify the 356 relative expression levels of the soybean and common bean genes from three biological replicates. 
357 All the primers used in this study are listed in Supplementary Table 11.

359 Gene set enrichment and pathway analysis. Gene ontology enrichment analysis of a given gene

360 group, such as, differentially expressed genes and mobile mRNAs was performed and visualized

361 with the clusterProfiler R package52 (version 3.10). The clusterProfiler's input files were formatted

362 using in-house Perl scripts. GO term information of soybean and common bean was extracted from

363 the gene annotation files in the Phytozome database. The GO terms, with FDR-adjusted $p$ value $<$

3640.05 , were considered as significant enrichment.

\section{Acknowledgements}

367 This work was partially supported by the Agriculture and Food Research Initiative of the USDA

368 National Institute of Food and Agriculture (2018-67013-27425), the National Natural Science

369 Foundation of China (31971898), the National Key R \& D Project of China (2016YFD0100304),

370 and the Purdue AgSEED program.

372 Author contributions

$373 \mathrm{JM}, \mathrm{SL}$ and XW designed the research; XW analyzed the data; SL, XW, WX, TL, CC, LC, and CC

374 performed the research; JM wrote the manuscript with input from SL and XW. 


\section{Conflicting interest}

377 The authors declare no conflict of interest.

378

379 Data availability

380 The raw read sequences are deposited in the National Center for Biotechnology Information

381 Sequence Read Achieve (http://www.ncbi.nlm.nih.gov/sra/) under the accession number

382 PRJNA648759.

383

$384 \quad$ References

385 1. Wang, J. \& Jiang, L., Wu, R. Plant grafting: how genetic exchange promotes vascular 386 reconnection. New Phytol 214, 56-65 (2017).

387 2. Kehr, J. \& Kragler, F. Long distance RNA movement. New Phytol 218, 29-40 (2018).

388 3. Liu, L. \& Chen, X. Intercellular and systemic trafficking of RNAs in plants. Nat Plants 4, $389 \quad 869-878(2018)$.

390 4. Lee, J.Y. Plasmodesmata: a signaling hub at the cellular boundary. Curr Opin Plant Biol $391 \quad 27,133-140(2015)$.

392 5. Aloni, B., Cohen, R., Karni, L., Aktas, H. \& Edelstein, M. Hormonal signaling in 393 rootstock-scion interactions. Sci Hortic 127, 119-126 (2010).

394 6. Zhang, S., Sun, L. \& Kragler, F. The phloem-delivered RNA pool contains small noncoding 
RNAs and interferes with translation. Plant Physiol 150, 378 (2009).

7. Thieme, C.J. et al. Endogenous Arabidopsis messenger RNAs transported to distant tissues. Nat Plants 1, 15025 (2015).

8. Ruiz-Medrano, R., Xoconostle-Cázares, B. \& Lucas, W.J. Phloem long-distance transport 399

400

401

402

403

404

405

406

407 408

409

410

411

412 of CmNACP mRNA: implications for supracellular regulation in plants. Development 126, 4405-4419 (1999).

9. Banerjee, A.K., Chatterjee, M., Yu, Y., Suhm S-G. \& Miller, W.A., Hannapelm D.J. Dynamics of a mobile RNA of potato involved in a long-distance signaling pathway. Plant Cell 18, 3443-3457 (2006).

10. Lu, K.J, Huang, N.C, Liu, Y.S, Lu, C.A. \& Yu, T.S. Long-distance movement of Arabidopsis FLOWERING LOCUS T RNA participates in systemic floral regulation. $R N A$ Biol 9, 653-662 (2012).

11. Mahajan, A., Bhogale, S., Kang, I.H., Hannapel, D.J. \& Banerjee, A.K. The mRNA of a Knotted1-like transcription factor of potato is phloem mobile. Plant Mol Biol 79, 595-608 (2012).

12. Notaguchi, M., Wolf, S. \& Lucas, W.J. Phloem-mobile Aux/IAA transcripts target to the root tip and modify root architecture. J Integr Plant Biol 54, 760-772 (2012).

13. Zhang, C. et al. Rhizobial infection triggers systemic transport of endogenous RNAs between shoots and roots in soybean. Sci China Life Sci 63, 1213-1226 (2020).

14. Yoo, B.C. et al. A systemic small RNA signaling system in plants. Plant Cell 16, 1979 (2004).

15. Buhtz, A., Springer, F., Chappell, L., Baulcombe, D.C., Kehr, J. Identification and characterization of small RNAs from the phloem of Brassica napus. Plant $J$ 53, 739-749 (2008). 
419 16. Pant, BD. et al. Identification of nutrient-responsive Arabidopsis and rapeseed microRNAs by comprehensive real-Time polymerase chain Reaction profiling and small RNA sequencing. Plant Physiol 150, 1541-1555 (2009).

422 17. Ham, B.K. \& Lucas, W.J. Phloem-mobile RNAs as systemic signaling agents. Annu Rev 423 Plant Biol 68, 173-195 (2017).

424 18. Lin, S.I. et al. Regulatory network of microRNA399 and PHO2 by systemic signaling. 425 Plant Physiol 147, 732 (2008).

426 19. Pant, B.D., Buhtz, A., Kehr, J. \& Scheible, W.R. MicroRNA399 is a long-distance signal for the regulation of plant phosphate homeostasis. Plant J 53, 731-738 (2008).

428 429 430 431 432 433 434 435 436

437 24. Vazquez, F. et al. Endogenous trans-acting siRNAs regulate the accumulation of 438

26. Chitwood, D.H., Nogueira, F.T.S., Howell, M.D., Montgomery, T.A., Carrington, J.C. \& 442 Arabidopsis mRNAs. Mol Cell 16, 69-79 (2004).

25. Allen, E., Xie, Z., Gustafson, A.M. \& Carrington, J.C. microRNA-directed phasing during trans-acting siRNA biogenesis in plants. Cell 121, 207-221 (2005).

Timmermans, M.C.P. Pattern formation via small RNA mobility. Genes Dev 23, 549-554 
444 27. Schmutz, J. et al. Genome sequence of the palaeopolyploid soybean. Nature 463, 178-183 (2010).

446 28. Schmutz, J. et al. A reference genome for common bean and genome-wide analysis of dual 447 domestications. Nat Genet 46, 707-713 (2014).

448 449 450

451

452 453

454

455 456

457 458 459

460 461 462 463 464 465 466

29. Melnyk, C.W., Molnar, A., Bassett, A. \& Baulcombe, D.C. Mobile 24 nt small RNAs direct transcriptional gene silencing in the root meristems of Arabidopsis thaliana. Curr Biol 21, 1678-1683 (2011).

30. Zhang, Z., Liu, X., Guo, X., Wang, X.J. \& Zhang, X. Arabidopsis AGO3 predominantly recruits 24-nt small RNAs to regulate epigenetic silencing. Nat Plants 2, 16049 (2016).

31. Zhai, J. et al. MicroRNAs as master regulators of the plant NB-LRR defense gene family via the production of phased, trans-acting siRNAs. Genes Dev 25, 2540-2553 (2011).

32. Boualem, A. et al. MicroRNA166 controls root and nodule development in Medicago truncatula. Plant J 54, 876-887 (2008).

33. Zhao, M., Meyers, B.C., Cai, C., Xu, W. \& Ma, J. Evolutionary patterns and coevolutionary consequences of MIRNA genes and microRNA targets triggered by multiple mechanisms of genomic duplications in soybean. Plant Cell 27, 546-562 (2015).

34. Fei, Q., et al. Biogenesis of a 22-nt microRNA in Phaseoleae species by precursorprogrammed uridylation. Proc Natl Acad Sci USA 115, 8037-8042 (2018).

35. Xia, C., Huang, J., Lan, H. \& Zhang, C. Long-distance movement of mineral deficiencyresponsive mRNAs in Nicotiana Benthamiana/tomato heterografts. Plants 9, 876 (2020).

36. Xia, C., et al. Elucidation of the mechanisms of long-distance mRNA movement in a Nicotiana benthamiana/tomato heterograft system. Plant Physiology 177, 745 (2018).

37. Langmead, B., Trapnell, C., Pop, M. \& Salzberg, S.L. Ultrafast and memory-efficient 
alignment of short DNA sequences to the human genome. Genome Biol 10, R25 (2009).

468 38. Dobin, A. et al. STAR: ultrafast universal RNA-seq aligner. Bioinformatics 29, 15-21

469 (2013).

470 39. Li, H. et al. The sequence alignment/map format and SAM tools. Bioinformatics 25, 20782079 (2009).

472 40. Quinlan, A.R. \& Hall, I.M. BEDTools: a flexible suite of utilities for comparing genomic features. Bioinformatics 26, 841-842 (2010).

474 41. Robinson, M.D., McCarthy, D.J. \& Smyth, G.K. edgeR: a Bioconductor package for 475 476

43. Arikit, S. et al. An atlas of soybean small RNAs identifies phased siRNAs from hundreds

42. Li, H. Minimap2: pairwise alignment for nucleotide sequences. Bioinformatics 34, 3094-

481

482

483

484 differential expression analysis of digital gene expression data. Bioinformatics 26, 139-140 (2009). 3100 (2018). of coding genes. Plant Cell 26, 4584-4601 (2014).

44. Thody, J., Folkes, L., Medina-Calzada, Z., Xu, P., Dalmay, T. \& Moulton, V. PAREsnip2: a tool for high-throughput prediction of small RNA targets from degradome sequencing data using configurable targeting rules. Nucleic Acids Res 46, 8730-8739 (2018).

45. Kozomara, A., Birgaoanu, M. \& Griffiths-Jones, S. miRBase: from microRNA sequences to function. Nucleic Acids Res 8, 155-162 (2019).

46. Guo, Q., Qu, X. \& Jin, W. PhaseTank: genome-wide computational identification of phasiRNAs and their regulatory cascades. Bioinformatics 31, 284-286 (2015).

47. Dai, X., Zhuang, Z. \& Zhao, P.X. psRNATarget: a plant small RNA target analysis server (2017 release). Nucleic Acids Res 46, W49-W54. (2018).

48. Ren, B., Wang, X., Duan, J. \& Ma, J. Rhizobial tRNA-derived small RNAs are signal 
molecules regulating plant nodulation. Science 365, 919-922 (2019).

492 49. Varkonyi-Gasic, E., Wu, R., Wood, M., Walton, E.F. \& Hellens, R.P. Protocol: a highly sensitive RT-PCR method for detection and quantification of microRNAs. Plant Methods 3, 12 (2007).

495 50. Libault, M. et al. Identification of four soybean reference genes for gene expression normalization. Plant Genome 1, 44-54 (2008).

497 51. Borges, A., Tsai, S.M. \& Caldas, D.G.G. Validation of reference genes for RT-qPCR 498 normalization in common bean during biotic and abiotic stresses. Plant Cell Rep 31, 827-

499 838 (2012).

500 52. Yu, G., Wang, L.G., Han, Y. \& He, Q.Y. clusterProfiler: an R package for comparing 501 biological themes among gene clusters. OMICS 16, 284-287 (2012).

502 
a

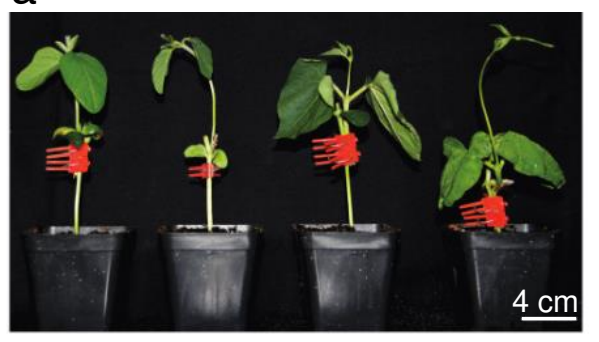

hoGmSt/ heGmSt/ hePvSt/ hoPvSt/ hoGmRt hePvRt heGmRt hoPvRt

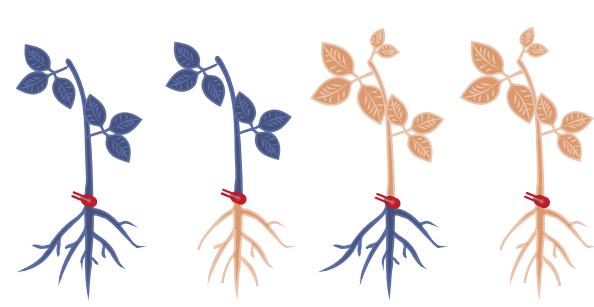

e

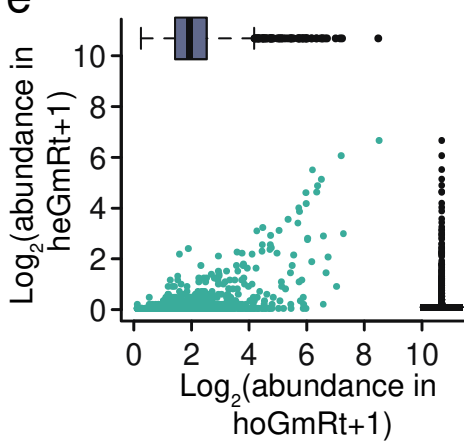

b

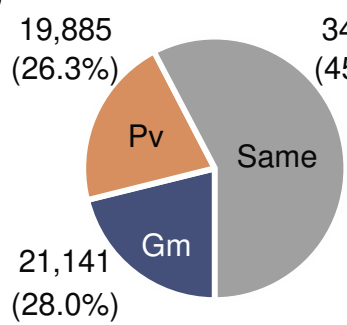

d

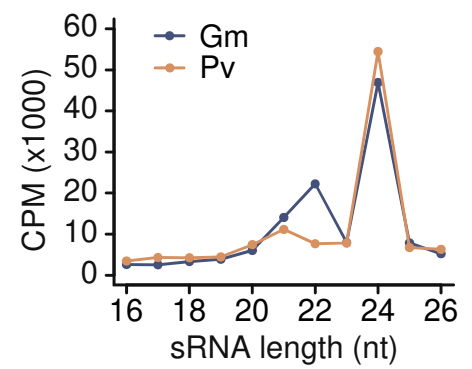

f

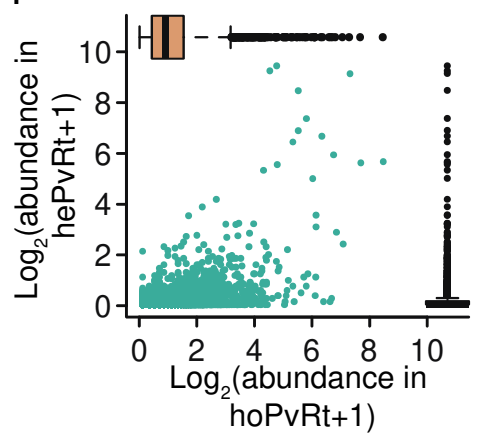

C

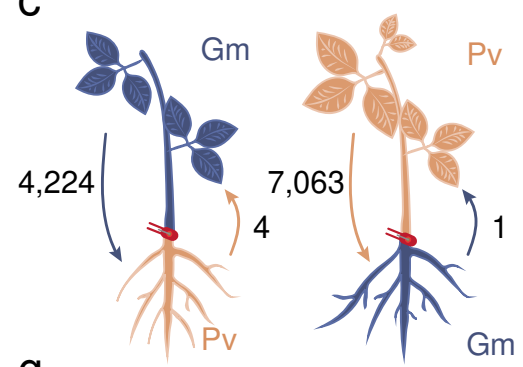

g

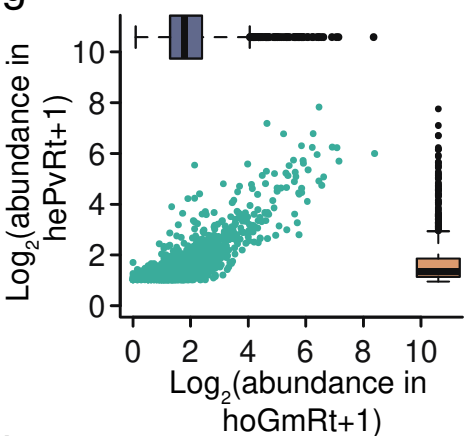

h

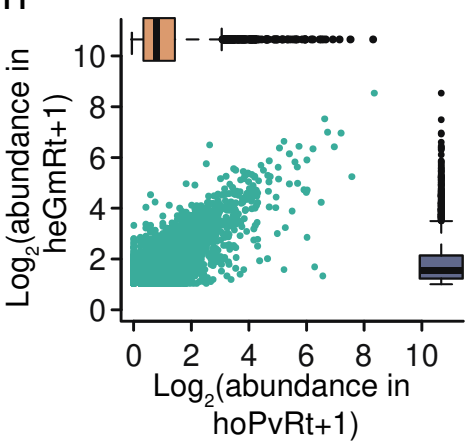

Figure 1. Mobility and relative abundance of soybean and common bean sRNAs. a. Images and schematic diagrams of the homograft and heterograft plants. The blue and orange colors indicate the soybean and common bean tissues, respectively. Codes for the eight samples are composed of the following abbreviations: "ho", homografted; "he" heterografted; "Gm", Glycine max (soybean); "Pv", Phaseolus vulgaris (common bean); "St" shoot; "Rt", root. b, Proportions of soybean and common bean sRNAs showing sequence variation and sRNAs showing "same" sequences between the two crops. c, Numbers of mobile soybean (blue) and common bean (orange) sRNAs and directionality of movement. d, Abundance of mobile soybean (Gm) and common bean (Pv) sRNAs ranging from 16-26 nt. e, Comparison of soybean hsciRNA abundances between heGmRt and hoGmRt. f, Comparison of common bean hsciRNA abundances between hePvRt and hoPvRt. g, Comparison of soybean hcsiRNA abundances between hePvRt and hoGmRt. h, Comparison of common bean hcsiRNA abundances between between heGmRt and hoPvRt. Boxplots display the distribution of the $\log 2$ transformed abundances of hcsiRNAs in individual samples. 
a

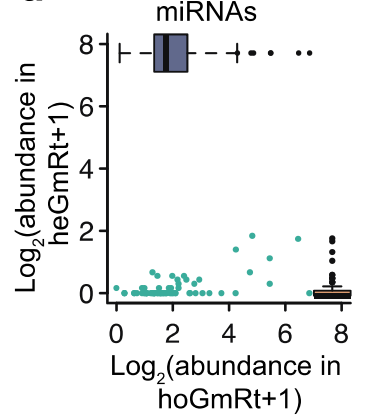

b

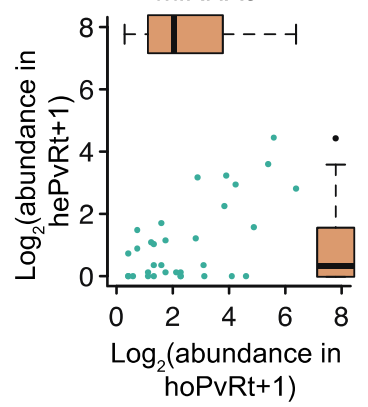

h
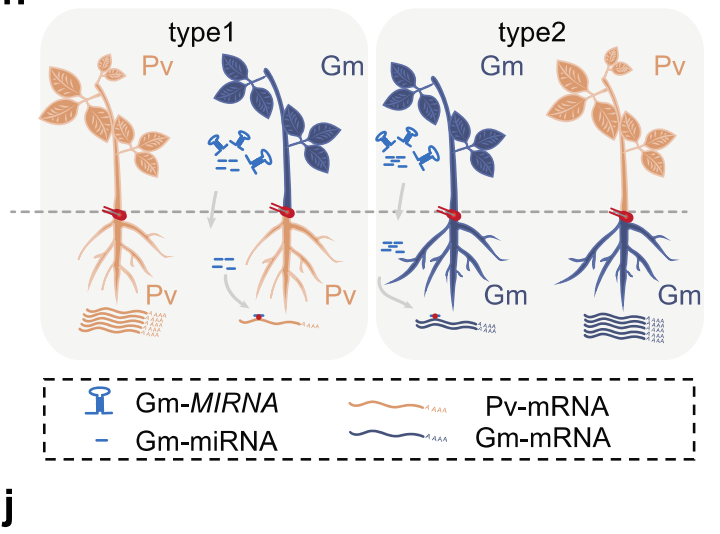

gma-miR4415a-3p 5' UUGAUUCUCAUCACAACAUGG 3'

Phvul.006G011700 3' AACUAAGGGUAGUGUGCACA 5' hePvRt: $30 / 46$ hoPvRt: 0/16

pvu-m0038 5' UUCAUUUUGAAAAUAGGCAUUG 3' \|\|\|\| 0 \|ा\|\|\|\|\|\|\|

Glyma.07G048100 3' AAGUAAAGAUUUUAUCCGUAAC 5' heGmRt: 26/53 hoGmRt: 0/17

gma-miR4996 5' UAGAAGCUCCCCAUGUUCUC 3' U

GLyma.13G183000 3' AUCUUGGAGGGGUACAGGAG 5' hoGmRt: $25 / 57 \Lambda_{\text {heGmRt: } 1 / 26}$

gma-miR4996 5' UAGAAGCUCCCCAUGUUCUC 3,

GLyma.13G183500 3' AUCUUGGAGGGGUACAGGAG 5' hoGmRt: 22/52 heGmRt: 0/24 e

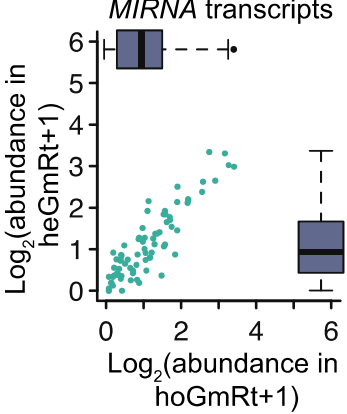

f hoGmRt+1)

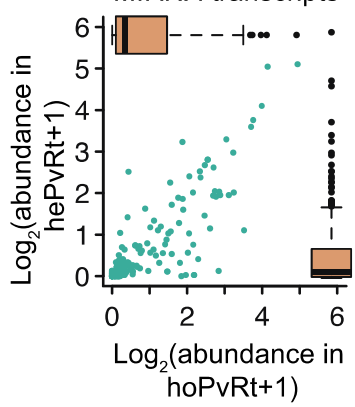

g

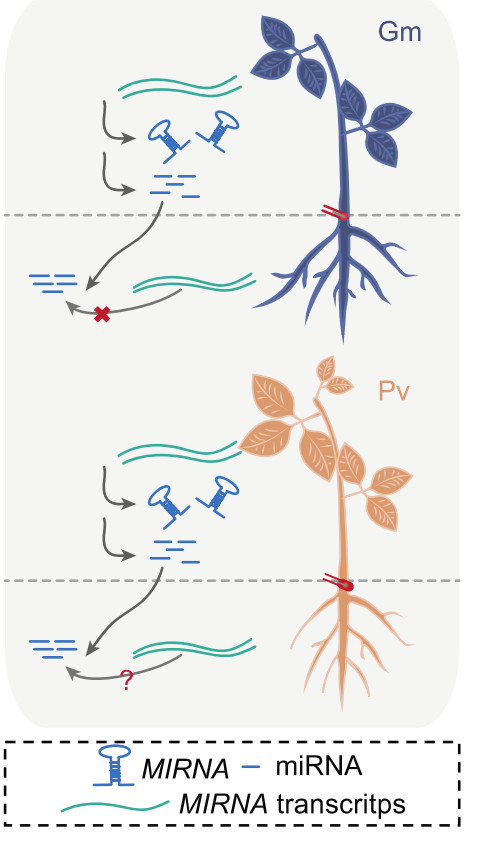

i

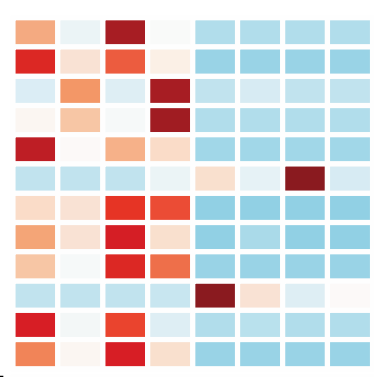

gma-miR4415a/b-3p gma-miR1507b

gma-miR4416b

gma-miR5770b

gma-miR5770a

pvu-m0038

gma-mIR4996

gma-miR1510b-3p

gma-miR2109-5p

pvu-m0053

gma-miR5376

gma-miR5767

Phvul.006G011700

Phvul.002G131200

Phvul.002G130300

Phvul.002G130600

Glyma.02G156800

Glyma.03G005400

Glyma.16G016600

Glyma.07G048100

Glyma.16G016700

Glyma.13G183000

Glyma.13G183500

Glyma.06G205100

Glyma.20G051900

Glyma.01G062400

Glyma.08G295100

Glyma.15G232400

Glyma.02G076700

Glyma.01G077900

Glyma.16G147100

Glyma.07G051600

Glyma.19G054900

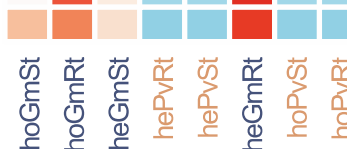

High

Low 
521 Figure 2. Relative abundance and systemic gene regulation of mobile miRNAs. a. Comparison

522 of soybean miRNA abundances between heGmRt and hoGmRt. b, Comparison of common bean

523 miRNA abundances between hePvRt and hoPvRt. c, Comparison of soybean miRNA abundances

524 between hePvRt and hoGmRt. $\mathbf{h}$, Comparison of common bean miRNA abundances between

525 between heGmRt and hoPvRt. e, Comparison of soybean MiRNA abundances between heGmRt

526 and hoGmRt. f, Comparison of common bean MiRNA abundances between hePvRt and hoPvRt.

527 Boxplots displayed the distribution of the $\log 2$ transformed abundances of miRNAs or MiRNAs in

528 individual samples. g, Modes of mobile miRNA biogenesis and systemic regulation of target genes.

$529 \mathbf{h}$ and $\mathbf{i}$, Exemplification of mobile miRNA-mediated down-regulation of target genes in recipient

530 tissues revealed by comparison of mRNA abundances between homografted and hetergrafted roots

531 of a same crop, e.g., down-regulation of Pv-mRNAs (or Gm-mRNAs) by mobile Gm-miRNAs (or

532 Pv-miRNAs) in hePvRt (or heGmRt) (type 1), and down-regulation of Gm-mRNAs by mobile

$533 \mathrm{Gm}-m i R N A s$ in hoGmRt (type 2). The curved lines with arrowheads connect miRNAs and

534 respective target genes. j, Exemplification of confirmed cleavages (sites and frequencies indicated

535 by arrows and ratios, respectively) of mRNAs by mobile miRNAs in recipient tissues of 536 hetergrafted and homografted plants. 


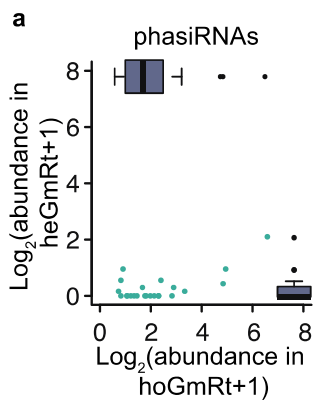

b phasiRnAs

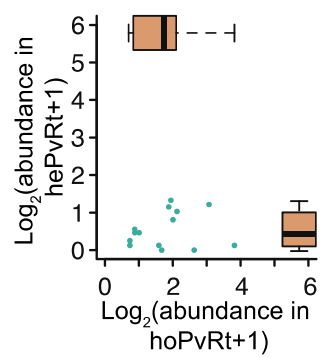

g

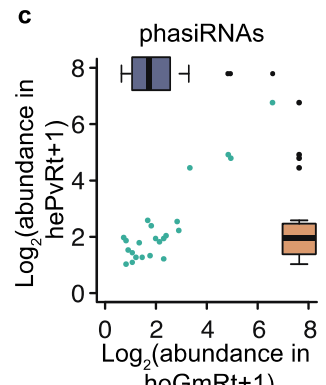

hoGmRt+1)

d phasiRnAs

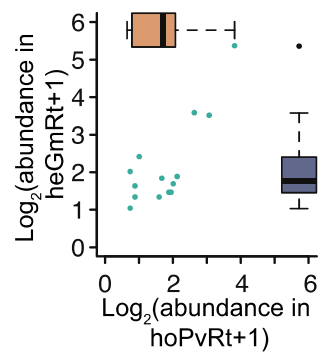

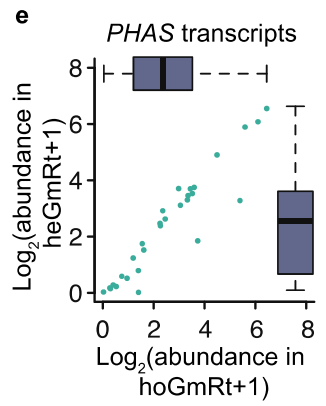

f

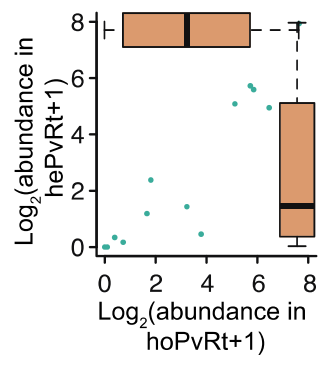

PHAS
537

538

539

540

541

542

543

544

545

546

547

548

549

550

551

552 h
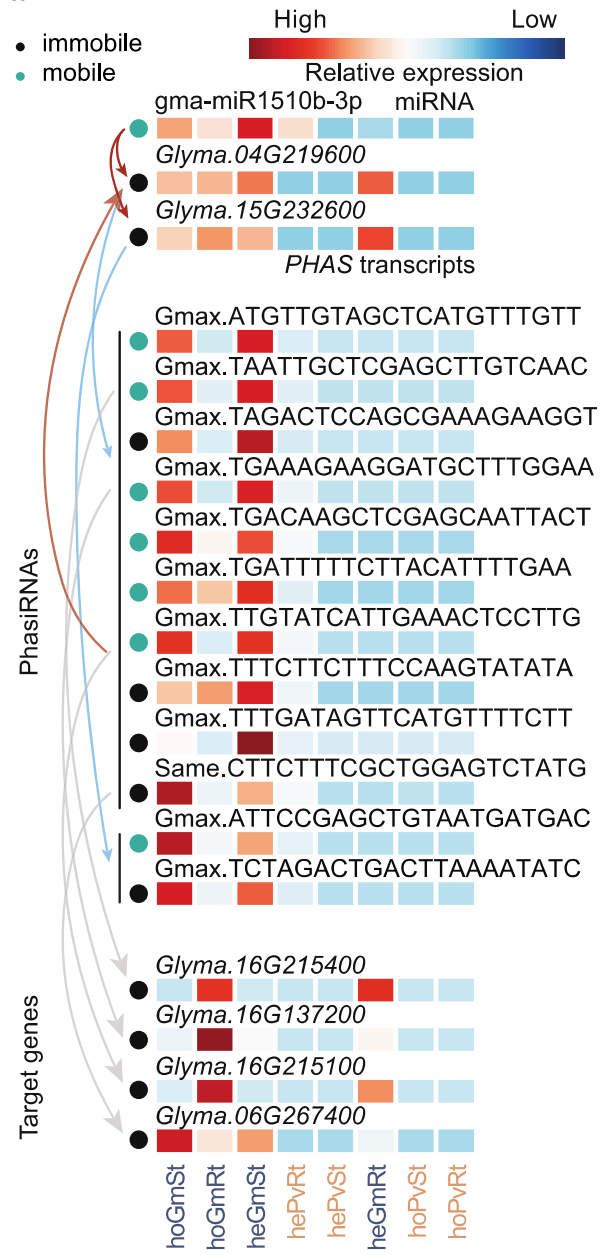

Figure 3. Relative abundance and systemic gene regulation of mobile phasiRNAs. a. Comparison of soybean phasiRNA abundances between heGmRt and hoGmRt. b, Comparison of common bean phasiRNA abundances between hePvRt and hoPvRt. c, Comparison of soybean phasiRNA abundances between hePvRt and hoGmRt. d, Comparison of common bean phasiRNA abundances between heGmRt and hoPvRt. e, Comparison of soybean PHAS transcript abundances between heGmRt and hoGmRt. f, Comparison of common bean PHAS transcript abundances between hePvRt and hoPvRt. Boxplots displayed the distribution of the log2 transformed abundances of phasiRNAs or PHAS transcripts in individual samples. g. Exemplification of regulatory cascades involving mobile phasiRNAs. gma-miR1510b-3p triggered production of a cluster of phasiRNAs from Glyma.04G219600 in soybean shoots, three of which enabled cleavage of their own precursor, with one validated to have implemented the cleavage in hoGmRt but not in heGmRt. Cleavage site and frequency are indicated by arrows and ratios, respectively. $\mathbf{h}$, Relative abundance of gma-miR1510b-3p, all phasiRNAs produced by the PHAS loci, and the $P H A S$ loci in the eight tissues. The curved lines with arrowheads connect the miRNA and its $P H A S$ targets, PHAS loci and phasiRNAs from the loci, or phasiRNAs and their respective target genes. 

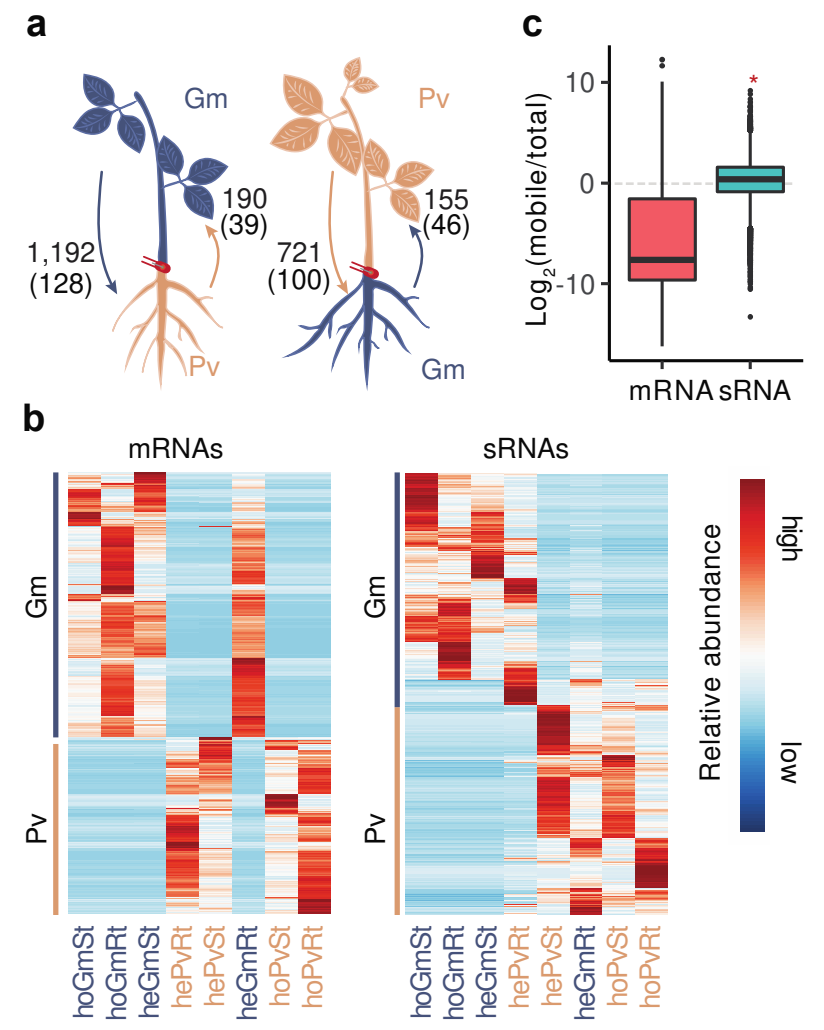

553

554

555

556

557

558

559

560

561

562

Figure 4. Differences in directionality and relative abundance between mobile sRNAs and mRNAs. a, Numbers of mobile soybean and common bean mRNAs detected by short-read Illumina RNA-seq (out of the brackets) and long-read Nanopore RNA-seq (within the brackets) and the directionality of mRNA movement. $\mathbf{b}$, Relative abundances of mobile mRNAs and sRNAs shown by heatmaps. The of blue and orange bars beside the heatmaps represent the RNAs from soybean $(\mathrm{Gm})$ and common bean $(\mathrm{Pv})$, respectively. c, Abundances of transported RNAs relative to abundances of respective RNAs accumulated (transported and locally produced) in recipient tissues shown by the distribution of the $\log 2$ transformed values. The grey dotted line indicates similar abundance between the transported RNAs in recipient tissues and locally produced RNAs. The red asterisk indicates significant difference ( $p$ value $<0.001, K-S$ test). 


\section{Figures}

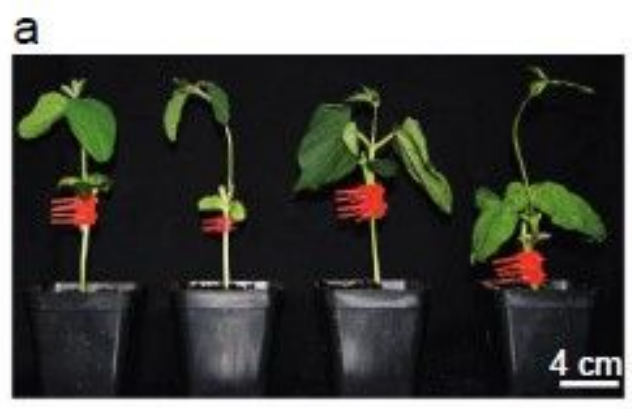

hoGmSt/ heGmSt/ hePvSt/ hoPvSt/ hoGmRt hePvRt heGmRt hoPvRt
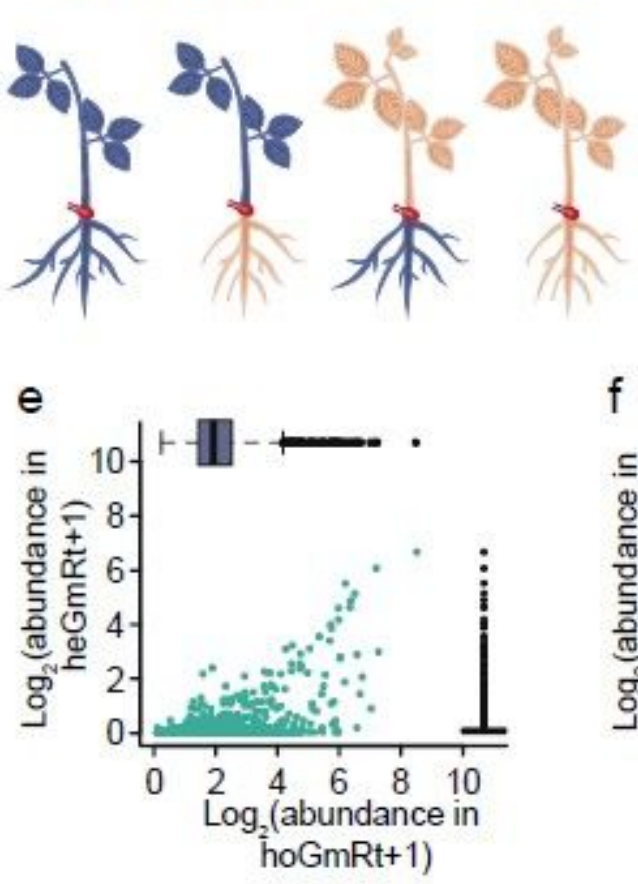

b

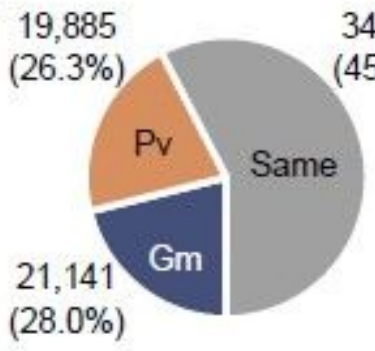

d

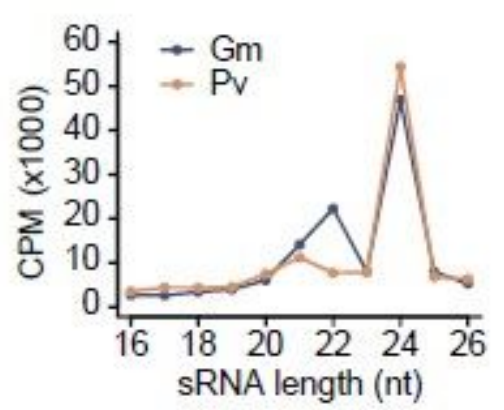

f

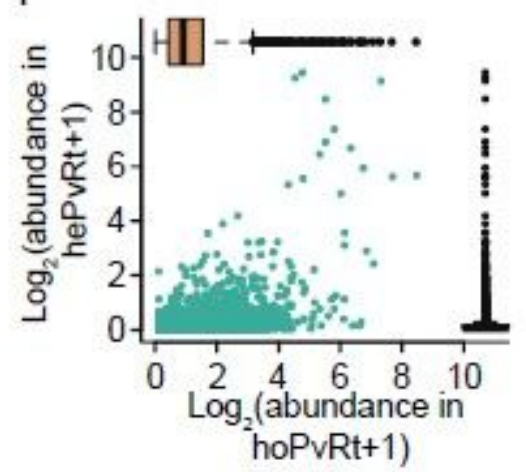

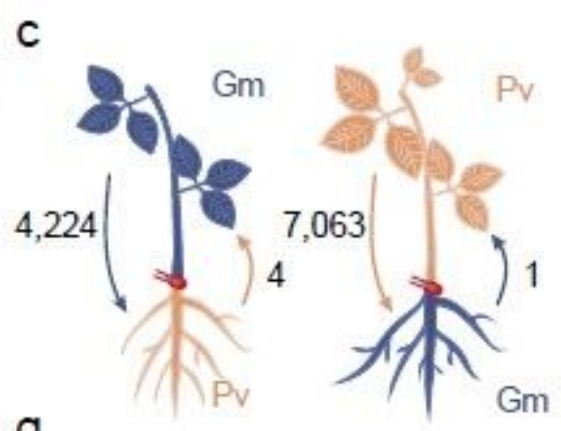

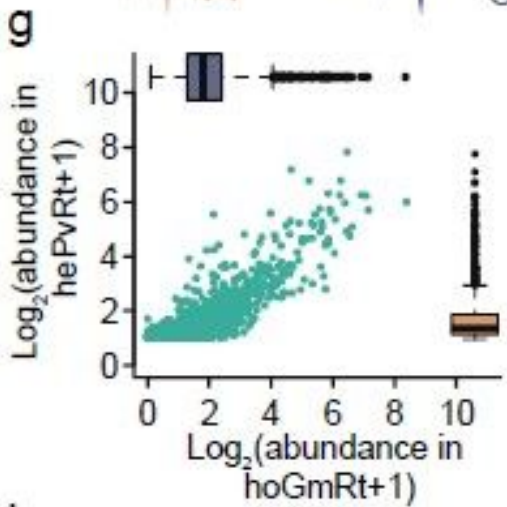

h

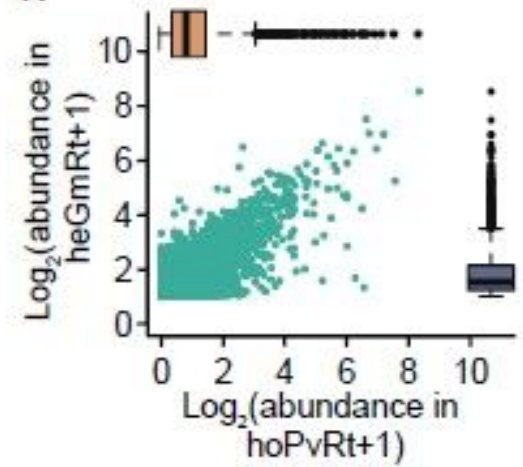

\section{Figure 1}

Mobility and relative abundance of soybean and common bean sRNAs. a. Images and schematic diagrams of the homograft and heterograft plants. The blue and orange colors indicate the soybean and common bean tissues, respectively. Codes for the eight samples are composed of the following abbreviations: "ho", homografted; "he" heterografted; "Gm”, Glycine max (soybean); "Pv", Phaseolus vulgaris (common bean); "St" shoot; "Rt", root. b, Proportions of soybean and common bean sRNAs showing sequence variation and sRNAs showing "same" sequences between the two crops. c, Numbers of mobile soybean (blue) and common bean (orange) sRNAs and directionality of movement. $d$, Abundance of mobile soybean $(\mathrm{Gm})$ and common bean $(\mathrm{Pv})$ sRNAs ranging from 16-26 nt. e, Comparison of soybean hsciRNA abundances between heGmRt and hoGmRt. f, Comparison of common bean hsciRNA abundances between hePvRt and hoPvRt. $\mathrm{g}$, Comparison of soybean hcsiRNA abundances between hePvRt and hoGmRt. h, Comparison of common bean hcsiRNA abundances between between 
heGmRt and hoPvRt. Boxplots display the distribution of the log2 transformed abundances of hcsiRNAs in individual samples

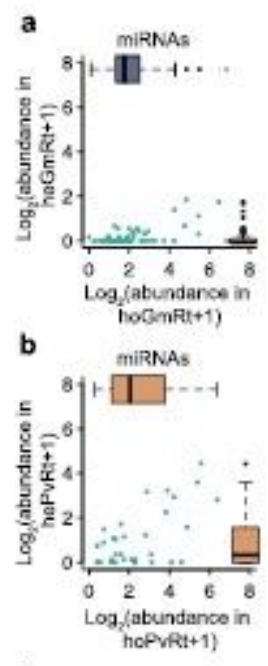

h

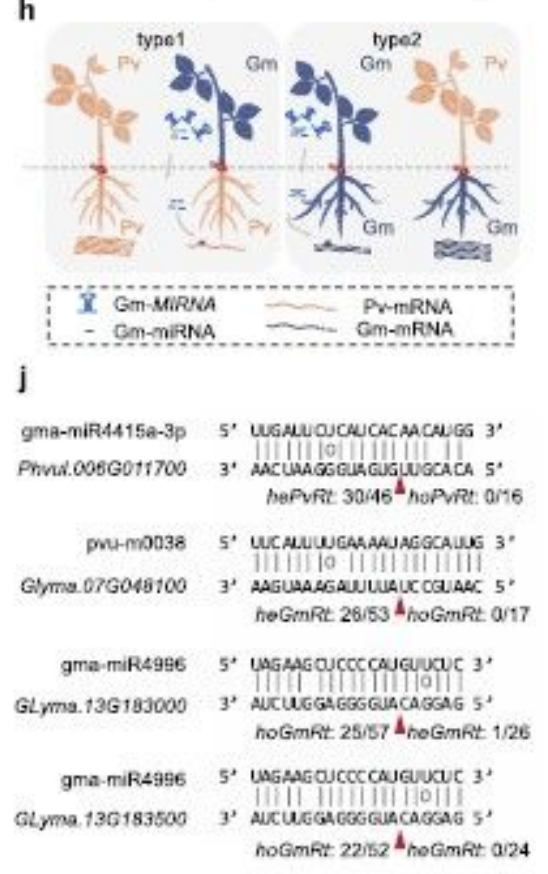

j
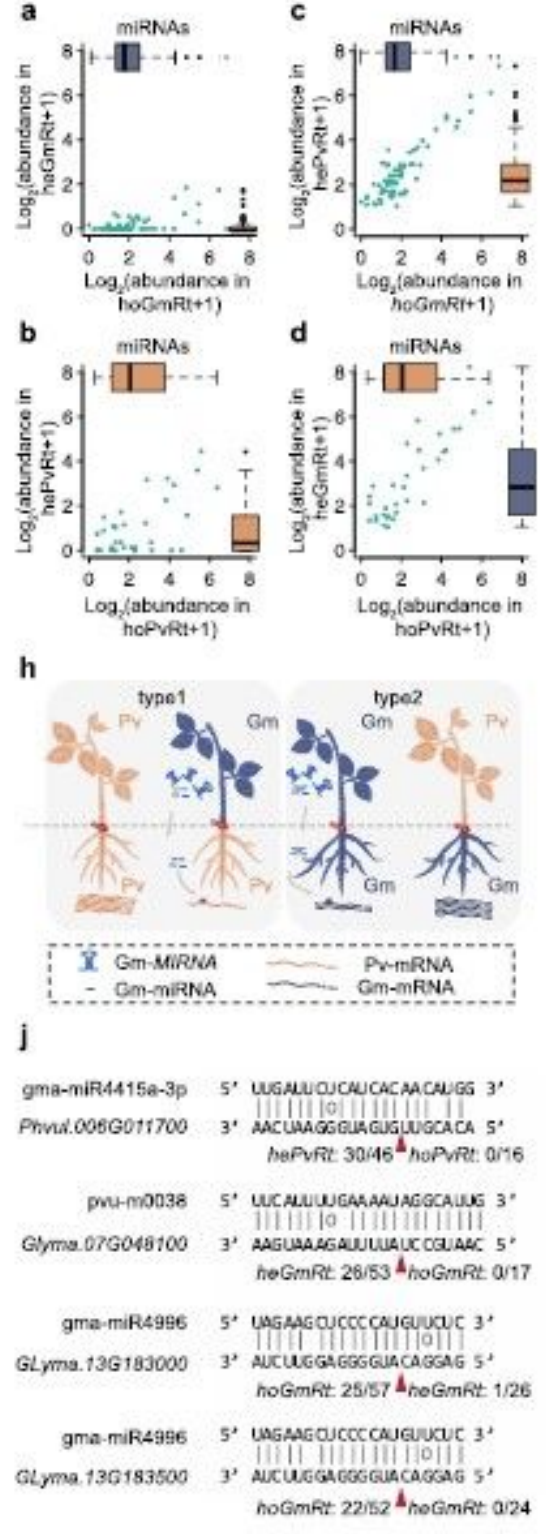
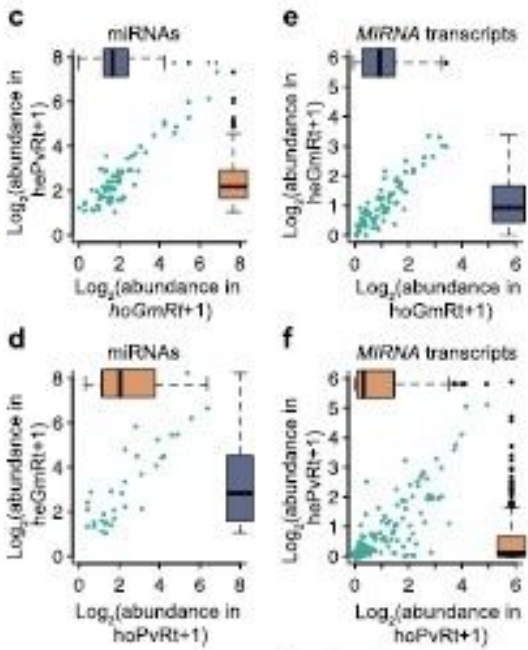

i

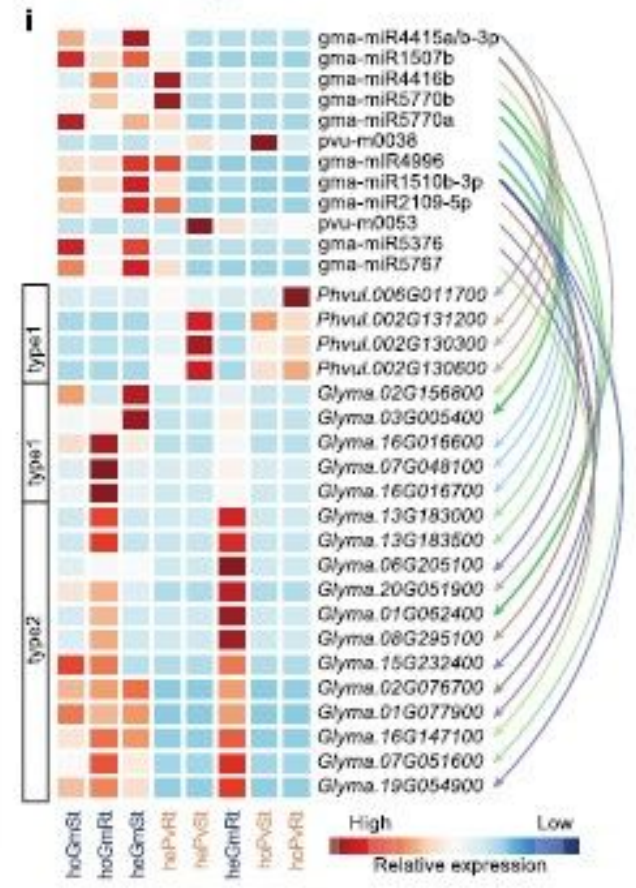

g
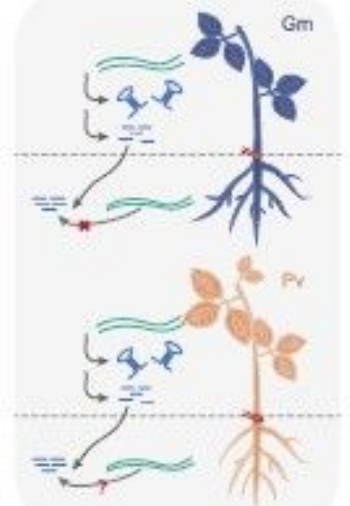

C.MiRA-makA

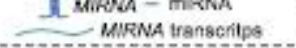

Figure 2

Relative abundance and systemic gene regulation of mobile miRNAs. a. Comparison of soybean miRNA abundances between heGmRt and hoGmRt. b, Comparison of common bean miRNA abundances between hePvRt and hoPvRt. c, Comparison of soybean miRNA abundances between hePvRt and hoGmRt. h, Comparison of common bean miRNA abundances between between heGmRt and hoPvRt. e, Comparison of soybean MiRNA abundances between heGmRt and hoGmRt. f, Comparison of common bean MiRNA abundances between hePvRt and hoPvRt. Boxplots displayed the distribution of the log2 transformed abundances of miRNAs or MiRNAs in individual samples. g, Modes of mobile miRNA biogenesis and systemic regulation of target genes. $h$ and $i$, Exemplification of mobile miRNA-mediated down-regulation of target genes in recipient tissues revealed by comparison of mRNA abundances between homografted and hetergrafted roots of a same crop, e.g., down-regulation of Pv-mRNAs (or Gm- 
mRNAs) by mobile Gm-miRNAs (or Pv-miRNAs) in hePvRt (or heGmRt) (type 1), and down-regulation of Gm-mRNAs by mobile Gm-miRNAs in hoGmRt (type 2). The curved lines with arrowheads connect miRNAs and respective target genes. j, Exemplification of confirmed cleavages (sites and frequencies indicated by arrows and ratios, respectively) of mRNAs by mobile miRNAs in recipient tissues of hetergrafted and homografted plants.

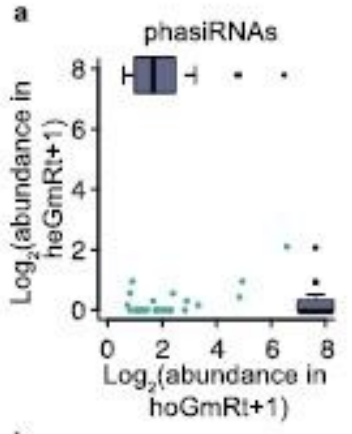

b

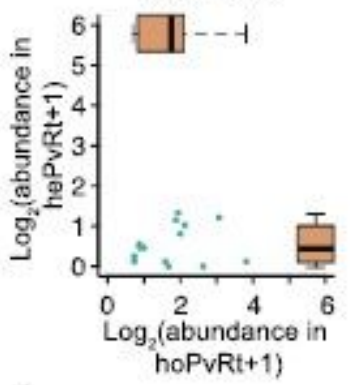

g

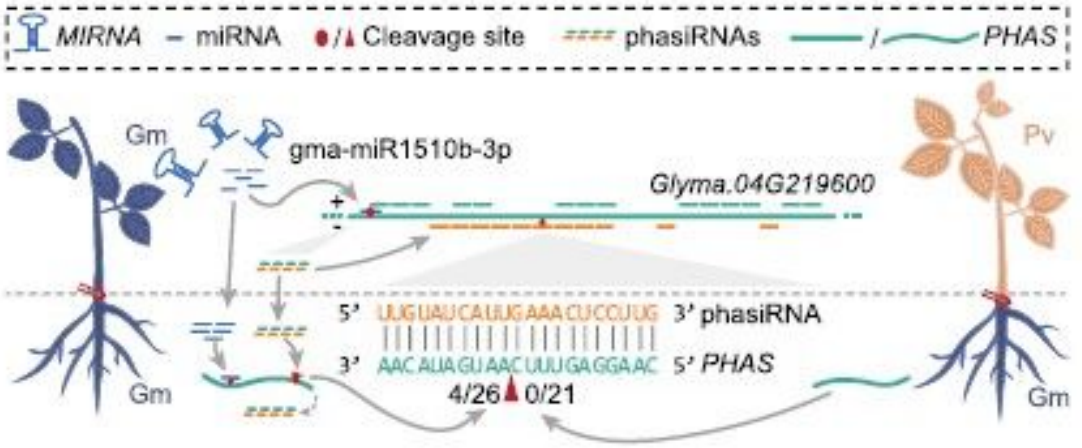

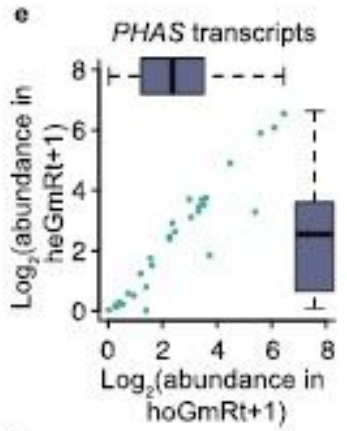

d

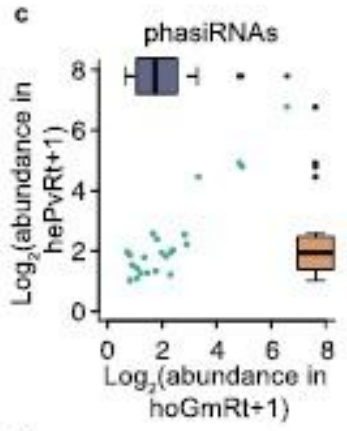

f

PHAS transcripts

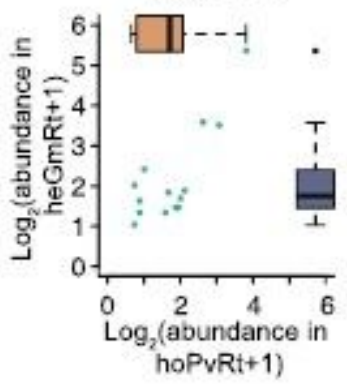

h

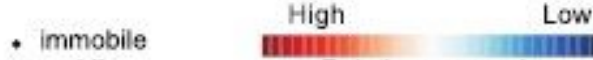

Relative expression

- mobile Ra-miR1510b-3p miRNA

Glyma.04G219600

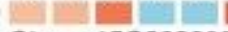

Glyms.15G232600

PHAS transcripts

Gmax.ATGTTGTAGCTCATGTTTGTT

Gmax.TAATTGCTCGAGCTTGTCAAC

Gmax.TAGACTCCAGCGAAAGAAGGT

Gmax. TGAAAGAAGgATGCTITGGAA

Gmax, TGACAAGCTCGAGCAATTACT

E D

Gmax.TGATITTTCTIACATIITGAA

Gmax.TTGTATCATTGAAACTCCTTG

Gmax.TTTCTTCTTTCCAAGTATATA

Gmax.TITgatagTtCATGTTTTCTT

Same.CTTCTTTCGCTGGAGTCTATG

Gmax.ATTCCGAGCTGTAATGATGAC

Gmax.TCTAGACTGACTTAAAATATC

Gmax. TCTAGACTGACTTAAA

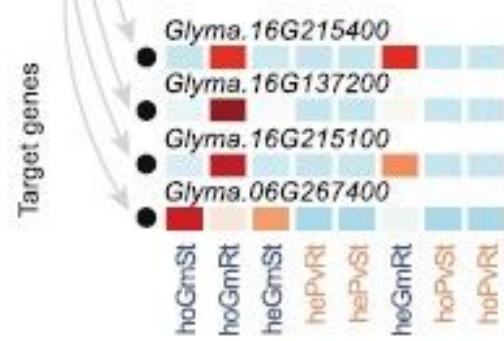

Figure 3

Relative abundance and systemic gene regulation of mobile phasiRNAs. a. Comparison of soybean phasiRNA abundances between heGmRt and hoGmRt. b, Comparison of common bean phasiRNA abundances between hePvRt and hoPvRt. c, Comparison of soybean phasiRNA abundances between hePvRt and hoGmRt. d, Comparison of common bean phasiRNA abundances between heGmRt and hoPvRt. e, Comparison of soybean PHAS transcript abundances between heGmRt and hoGmRt. f, Comparison of common bean PHAS transcript abundances between hePvRt and hoPvRt. Boxplots displayed the distribution of the log2 transformed abundances of phasiRNAs or PHAS transcripts in individual samples. g. Exemplification of regulatory cascades involving mobile phasiRNAs. gmamiR1510b-3p triggered production of a cluster of phasiRNAs from Glyma.04G219600 in soybean shoots, three of which enabled cleavage of their own precursor, with one validated to have implemented the 
cleavage in hoGmRt but not in heGmRt. Cleavage site and frequency are indicated by arrows and ratios, respectively. h, Relative abundance of gma-miR1510b-3p, all phasiRNAs produced by the PHAS loci, and the PHAS loci in the eight tissues. The curved lines with arrowheads connect the miRNA and its PHAS targets, PHAS loci and phasiRNAs from the loci, or phasiRNAs and their respective target genes.
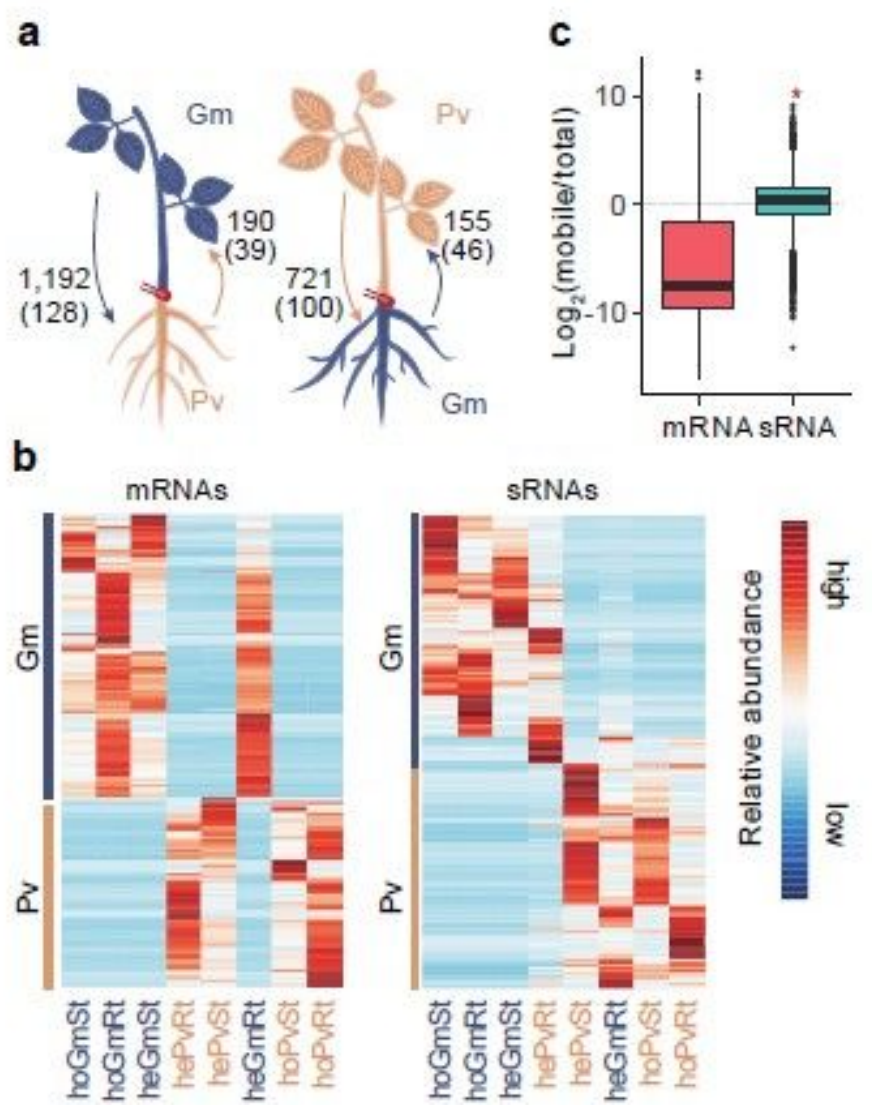

\section{Figure 4}

Differences in directionality and relative abundance between mobile sRNAs and mRNAs. a, Numbers of mobile soybean and common bean mRNAs detected by short-read Illumina RNA-seq (out of the brackets) and long-read Nanopore RNA-seq (within the brackets) and the directionality of mRNA movement. b, Relative abundances of mobile mRNAs and sRNAs shown by heatmaps. The of blue and orange bars beside the heatmaps represent the RNAs from soybean $(\mathrm{Gm})$ and common bean (Pv), respectively. $\mathrm{c}$, Abundances of transported RNAs relative to abundances of respective RNAs accumulated (transported and locally produced) in recipient tissues shown by the distribution of the log2 transformed values. The grey dotted line indicates similar abundance between the transported RNAs in recipient tissues and locally produced RNAs. The red asterisk indicates significant difference ( $p$ value $<0.001$, K-S test).

\section{Supplementary Files}

This is a list of supplementary files associated with this preprint. Click to download.

- TableS1.png 
- SupplementaryTable2.xlsx

- SupplementaryTable3.xlsx

- SupplementaryTable4.xIsx

- SupplementaryTable5.xIsx

- SupplementaryTable6.xIsx

- SupplementaryTable7.xlsx

- SupplementaryTable8.xlsx

- SupplementaryTable9.xlsx

- SupplementaryTable10.xIsx

- SupplementaryTable11.xlsx

- SupplementaryDataset1.xlsx

- FigureS1.jpg

- FigureS2.jpg

- FigureS3.jpg 